Research Notes

\title{
Subcellular Localization Studies of Three Phenylalanine Ammonia-Lyases and Cinnamate 4-Hydroxylase from Scutellaria Baicalensis Using GFP Fusion Proteins
}

\author{
${ }^{1}$ Nam Il Park, ${ }^{2}$ Hui Xu, ${ }^{3}$ Mariadhas Valan Arasu, ${ }^{3}$ Naif Abdullah Al-Dhabi and ${ }^{4,5}$ Sang Un Park \\ ${ }^{1}$ Department of Plant Science, Gangeung-Wonju National University, 7 Jukheon-gil, Gangneung-si, \\ Gangwon-do 210-702, Korea \\ ${ }^{2}$ College of Life Science, Inner Mongolia University for Nationalities, Inner Mongolia, China \\ ${ }^{3}$ Department of Botany and Microbiology, Addiriyah Chair for Environmental Studies, College of Science, \\ King Saud University, P. O. Box 2455, Riyadh 11451, Saudi Arabia \\ ${ }^{4}$ Department of Crop Science, Chungnam National University, 99 Daehak-ro, Yuseong-gu, Daejeon, 305-764, Korea \\ ${ }^{5}$ Visiting Professor Program (VPP), King Saud University, P.O. Box 2455, Riyadh 11451, Saudi Arabia
}

\author{
Article history \\ Received: 28-03-2015 \\ Revised: 16-04-2015 \\ Accepted: 01-06-2015 \\ Corresponding Author: \\ Sang Un Park \\ Department of Crop Science, \\ Chungnam National \\ University, 99 Daehak-ro, \\ Yuseong-gu, Daejeon, 305- \\ 764 , Korea \\ Tel: +82428215730 , \\ Fax: +82428222631 \\ Email: supark@cnu.ac.kr
}

\begin{abstract}
The localization of three phenylalanine ammonia-lyases (PAL1, 2 and -3$)$ and Cinnamate 4-Hydroxlase $(\mathrm{C} 4 \mathrm{H})$ of Scutellaria baicalensis was examined in onion epidermal cells. These genes encode key enzymes in the phenylpropanoid pathway for the synthesis of flavones. In our previous research, we isolated coding DNA for these genes from $S$. baicalensis, a medicinal herb rich in flavones with biological and pharmacological properties. We observed that SbPAL2, SbPAL3 and SbC4H proteins localize to the endoplasmic reticulum; however, SbPAL1 was a cytosolic protein. Unlike SbPAL2 and SbPAL3, SbPAL1 may be expected to have a different function in the flavone biosynthetic pathway.
\end{abstract}

Keywords: Cinnamate 4-Hydroxylase, GFP, Phenylalnine AmmoniaLyases, Scutellaria Baicalenesis

\section{Introduction}

Scutellaria baicalensis Georgi, golden root, is a species of flowering plant in the Lamiaceae family. It is one of the 50 fundamental herbs used in traditional Chinese medicine and is prescribed for fever, cold and hematemesis. In Western medicine, it has been used to treat inflammation, respiratory tract infections, diarrhea, dysentery, jaundice/liver disorders, hypertension, hemorrhaging and insomnia (Li et al., 2000; Nishikawa et al., 1999). Root of $S$. baicalensis is rich in flavones, a class of flavonoids produced by plants. These compounds exhibit biological and pharmacological properties, including antioxidative activity, cancer prevention and the treatment and prevention of coronary heart disease (Martens and Mithöfer, 2005).

Baicalin, baicalein and wogonin are the most well studied flavone constituents of $S$. baicalensis (Gao et al., 2000; Horvath et al., 2005). They provide a variety of health benefits; wogonin, especially, is a natural neuroprotective compound that inhibits the inflammatory response of microglia (Lee et al., 2003). S. baicalensis contains high concentrations of melatonin, a potent antioxidant (Murch et al., 2004). The main flavone compounds of $S$. baicalensis represent important sources for the development of anti-cancer, anti-inflammatory and neuroprotective drugs.

Phenylalanine ammonia-lyase (PAL, EC 4.3.1.5), the first key enzyme of the phenylpropanoid pathway, catalyzes the conversion of ${ }_{\mathrm{L}}$-phenylalanine to transcinnamic acid. PAL has been extensively studied in plants because of its important role in the biosynthesis of various secondary metabolites. PAL is thought to be responsible for many essential functions, including the establishment of mechanical support, the generation of pigments such as anthocyanins and signaling during flavonoid nodulation (Weisshaar and Jenkins, 1998). PAL also induces phenylpropanoid biosynthesis in response to biotic and abiotic stressors, such as pathogen attacks, UV irradiation, mechanical wounding and light (Dixon and Paiva, 1995).

Cinnamate 4-hydroxylase (C4H, EC 1.14.13.11), the second key enzyme of the phenylpropanoid pathway, catalyzes the hydroxylation of trans-cinnamic acid to $p$ coumaric acid (Russel, 1971). C4H constitutes the CYP73 family of the cytochrome P450 monooxygenases 
that catalyze monooxygenase reactions in plants that are often involved in the biosynthesis of diverse metabolites (Chapple, 1998). C4H controls the carbon flux required for the synthesis of many phytoalexins when plants are challenged by pathogens (Teutsch et al., 1993). Therefore, PAL and $\mathrm{C} 4 \mathrm{H}$ are important compounds involved in the regulation of biosynthesis and flux control in the $S$. baicalensis flavone pathway (Fig. 1a).

\section{Materials and Methods}

\section{Gene Cloning and Construction}

Full-length $S$. baicalensis PALs (SbPAL1, SbPAL2 and SbPAL3) and C4H (SbC4H) (GenBank accession numbers HM062775, HM062776, HM062777 and HM062778, respectively) were cloned by using rapid amplification of cDNA ends (RACE)-Polymerase Chain Reaction (PCR). SbPAL1, -2, -3 and SbC4H constructs fused with GFP for transient expression were generated by cloning Reverse Transcription (RT)-PCR products corresponding to the coding sequence for each gene into the $\mathrm{pENTR/D-TOPO}$ vector (Invitrogen). These were then recombined into a pK7FWG2 destination vector (obtained from the Functional Genomics Unit of the Department of Plant System Biology, VIB-Ghent University, The Netherlands) using Gateway LR Clonase II Plus Enzyme Mix (Invitrogen).

\section{GFP Analysis}

The GFP fusion proteins were transiently expressed following the biolistic transformation of onion (Allium сера) epidermal cells as previously described (Park and Muench, 2007). Briefly, $5 \mu \mathrm{g}$ of DNA was vigorously mixed with tungsten particles in a solution containing 1 $\mathrm{M} \mathrm{CaCl} 2$ and $16 \mathrm{mM}$ spermidine. After being washed with $70 \%$ and $95 \%$ ethanol, the DNA-coated particles were loaded onto macrocarriers and bombarded into onion epidermal cells using a particle gun (PDS-1000; Bio-Rad). Peeled epidermal cell layers were mounted on microscope slides in MS medium, covered with a cover glass and observed using a UPlanFL N $40 \times$ objective lens attached to a fluorescence microscope (BX51; Olympus, Tokyo, Japan). Images were captured using a DP71 microscope digital camera (Olympus).

\section{Results}

Green Fluorescent Protein (GFP) imaging has been widely used to study plant cells despite early problems with the use of the jellyfish GFP gene due to the production and distribution of modified, thermostable, highly fluorescent GFP molecules suitable for expression in plant cells (Haseloff and Siemering, 1998). GFP coding sequences have been fused at either the $5^{\prime}$ or $3^{\prime}$ end of the coding region of a DNA sequence of interest, leading to the production of $\mathrm{N}$ or C-terminal GFP fusion proteins, respectively.
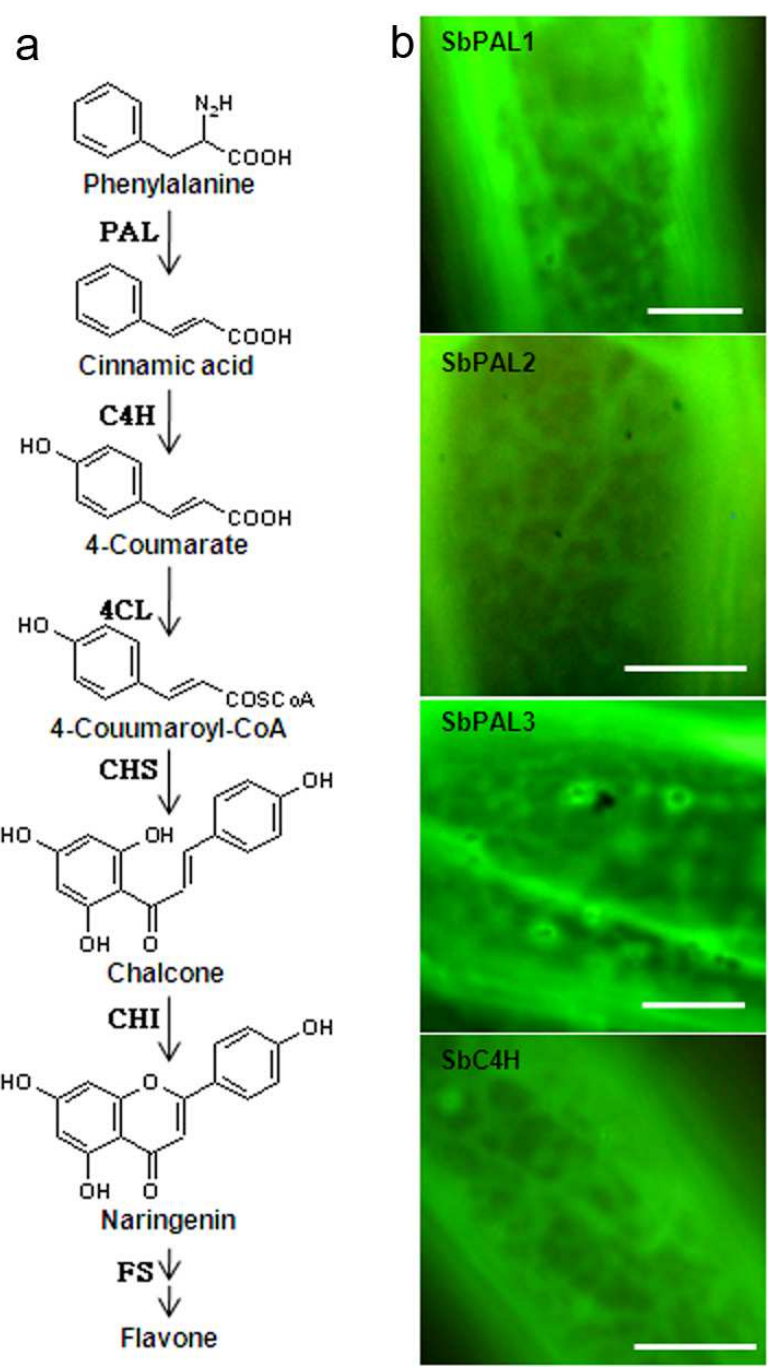

Fig. 1. The biosynthetic pathway of flavones and the subcellular localization of PALs and $\mathrm{C} 4 \mathrm{H}$. (a) The proposed pathway enzymes for the production of flavones include PAL, phenylalanine ammonia lyase; C4H, cinnamate 4-hydroxylase; 4CL, 4-coumarate: CoAligase; CHS, chalcone synthase; and FS, flavone synthase. (b) Subcellular localization of SbPAL1, -2 and -3 and $\mathrm{SbC} 4 \mathrm{H}$ in onion epidermal cells. Bars $=25 \mu \mathrm{m}$

GFP fusion genes have been used to produce stable transgenic plants or for their transient expression in plant cells. Onion epidermal cells are particularly useful material for transient expression assays because they have an exceptionally clear cytoplasm and consist of a single layer of living cells. The biolistic transformation of plant cells with various GFP fusion protein-expressing constructs has been used to visualize the cell wall, chloroplasts, cytoplasm, nucleus and Endoplasmic Reticulum (ER) (Scott et al., 1999).

The transient expression of GFP-fused SbPAL2, SbPAL3 and SbC4H resulted in a reticulate distribution 
of fluorescence in the examined cells; however, SbPAL1 localization was entirely cytosolic (Fig. 1b). Fluorescence from GFP only was observed throughout the cytoplasm and nucleus (data not shown).

\section{Discussion}

PAL and $\mathrm{C} 4 \mathrm{H}$ participate in the first and second steps of the phenylpropanoid pathway, respectively. $\mathrm{C} 4 \mathrm{H}$ anchors the enzyme complex, formed by the general phenylpropanoid enzymes, to the ER membrane (Winkel-Shirley, 1999). The ER localization of C4H has previously been shown in the hybrid poplar (Ro et al., 2001). In addition, tobacco PAL- and C4H-GFP fusion proteins localize to the ER and their co-localization was confirmed by using dual-labeling immunofluorescence and fluorescence resonance energy transfer studies (Achnine et al., 2004). Recent evidence that the other flavonoid enzymes, such as Chalcone Synthase (CHS) and Chalcone Isomerase (CHI), are localized in $A$. thaliana in the nucleus, suggests that flavonoid regulation of transcription is developmentally regulated at the subcellular level (Saslowsky et al., 2005).

Although our experiment did not show direct evidence that PALs co-localized with ER, the transient expression of GFP-fused SbPAL2, SbPAL3 and SbC4H resulted in a reticulate distribution of fluorescence in the examined cells. Unlike SbPAL2 and SbPAL3, however, SbPAL1 localization was entirely cytosolic. This result may be related that SbPAL1 formed a distinct group with SbPAL2 and SbPAL3 in a phylogenetic tree constructed from the deduced amino acid sequences of plant PALs (Xu et al., 2010).

\section{Conclusion}

Using in vivo localization technique, the results of this study indicate that SbPAL2, SbPAL3 and SbC4H proteins possible localize to the ER; however, SbPAL1 was a cytosolic protein. Unlike SbPAL2 and SbPAL3, SbPAL1 may be expected to have a different function in the flavone biosynthetic pathway.

\section{Acknowledgment}

The author Sang Un Park thanks the visiting professor program, Deanship of Scientific Research at King Saud University, Riyadh, Saudi Arabia.

\section{Funding Information}

The authors thank Addiriyah Chair for Environmental Studies, Department of Botany and Microbiology, College of Science, King Saud University, Saudi Arabia for the support.

\section{Author's Contributions}

Nam Il Park: Wrote the manuscript, performed the experiments and analyzed the data.

Hui Xu: Performed the experiments and analyzed the data.

Mariadhas Valan Arasu: Wrote the manuscript and analyzed the data.

Naif Abdullah Al-Dhabi: Review the manuscript and analyzed the data.

Sang Un Park: Designed the experiments and analyzed the data.

\section{Ethics}

The authors declare that there is no conflict of interests regarding the publication of this paper.

\section{References}

Achnine, L., E.B. Blancaflor, S. Rasmussen and R.A. Dixon, 2004. Colocalization of L-Phenylalanine ammonia-lyase and cinnamate 4-hydroxylase for metabolic channeling in phenylpropanoid biosynthesis. Plant Cell, 16: 3098-3109.

DOI: $10.1105 /$ tpc. 104.024406

Chapple, C., 1998. Molecular-genetic analysis of plant cytochrome P450dependent monooxygenases. Ann. Rev. Plant Physiol. Plant Mol. Biol., 49: 311-343. DOI: 10.1146/annurev.arplant.49.1.311

Dixon, R. and N. Paiva, 1995. Stress-induced phenylpropanoid metabolism. Plant Cell, 7: 1085-1097. DOI: 10.2307/3870059

Gao, Z., X. Yang, K. Huang and H. Xu, 2000. Freeradical scavenging and mechanism study of flavonoids extracted from the radix of Scutellaria baicalensis Georgi. Appl. Magn. Reson., 19: 35-44. DOI: $10.1007 /$ BF03162259

Haseloff, J. and K.R. Siemering, 1998. The Uses Of Gfp In Plants. In: Green Fluorescent Protein: Strategies, Applications Protocols, Chalfie M. and S. Kain, (Eds.), Wiley, pp: 191-220

Horvath, C.R., P.A. Martos and P.K. Saxena, 2005. Identification and quantification of eight flavones in root and shoot tissues of the medicinal plant Huang-qin (scutellaria baicalensis georgi) using highperformance liquid chromatography with diode array and mass spectrometric detection. J. Chromatogr. A., 1062: 199-207. DOI: 10.1016/j.chroma.2004.11.030

Lee, H., Y.O. Kim, H. Kim, S.Y. Kim and H.S. Noh et al., 2003. Flavonoid wogonin from medicinal herb is neuroprotective by inhibiting inflammatory activation of microglia. FASEB J., 17: 1943-1944. DOI: $10.1096 /$ fj.03-0057fje 
Li, H., S. Murch and P. Saxena, 2000. Thidiazuroninduced de novo shoot organogenesis on seedlings, etiolated hypocotyls and stem segments of Huangqin. Plant Cell, Tissue Organ Culture, 62: 169-173. DOI: $10.1023 / \mathrm{A}: 1006491408762$

Martens, S. and A. Mithöfer, 2005. Flavones and flavone synthases. Phytochemistry, 66: 2399-2407. DOI: 10.1016/j.phytochem.2005.07.013

Murch, S.J., H.P.V. Rupasinghe, D. Goodenowe and P.K. Saxena, 2004. A metabolomic analysis of medicinal diversity in Huang-qin (Scutellaria baicalensis georgi) genotypes: Discovery of novel compounds. Plant Cell Rep., 23: 419-425. DOI: 10.1007/s00299-004-0862-3

Nishikawa, K. and H. Furukawa, T. Fujioka, H. Fujii and K. Mihashi et al., 1999. Flavone production in transformed root cultures of Scutellaria baicalensis Georgi. Phytochemistry, 52: 885-890. DOI: 10.1016/S0031-9422(99)00306-4

Park, N.I. and D. Muench, 2007. Biochemical and cellular characterization of the plant ortholog of PYM, a protein that interacts with the exon junction complex core proteins Mago and Y14. Planta., 225: 625-639. DOI: $10.1007 / \mathrm{s} 00425-006-0385-\mathrm{y}$

Ro, D.K., N. Mah, B.E. Ellis and C.J. Douglas, 2001. Functional characterization and subcellular localization of poplar (Populus trichocarpa $\times$ Populus deltoides) cinnamate 4-hydroxylase. Plant Physiol., 126: 317-329. DOI: 10.1104/pp.126.1.317

Russel, D.W., 1971. The metabolism of aromatic compounds in higher plants. X. Properties of the cinnamic acid 4-hydroxylase of pea seedlings and some aspects of its metabolic and developmental control. J. Biol. Chem., 246: 3870-3878.
Saslowsky, D.E., U. Warek and B.S.J. Winkel, 2005. Nuclear localization of flavonoid enzymes in arabidopsis. J. Biol. Chem., 280: 23735-23740. DOI: $10.1074 /$ jbc.M413506200

Scott, A., S. Wyatt, P.L. Tsou, D. Robertson and N.S. Allen, 1999. Model system for plant cell biology: GFP imaging in living onion epidermal cells. Biotechniques, 26: 1125-1132. PMID: 10376152

Teutsch, H.G., M.P. Hasenfratz, A. Lesot, C. Stoltz and J.M. Garnier et al., 1993. Isolation and sequence of a cDNA encoding the Jerusalem artichoke cinnamate 4hydroxylase, a major plant cytochrome P450 involved in the general phenylpropanoid pathway. Proc. Natl. Acad. Sci. USA. 90: 4102-4106.

Weisshaar, B. and G.I. Jenkins, 1998. Phenylpropanoid biosynthesis and its regulation. Curr. Opin. Plant Biol., 1: 251-257. DOI: 10.1016/S1369-5266(98)80113-1

Winkel-Shirley, B., 1999. Evidence for enzyme complexes in the phenylpropanoid and flavonoid pathways. Physiol. Plant, 107: 142-149. DOI: 10.1034/j.1399-3054.1999.100119.x

Xu, H., N.I. Park, X. Li, Y.K. Kim and S.Y. Lee et al., 2010. Molecular cloning and characterization of phenylalanine ammonia-lyase, cinnamate 4hydroxylase and genes involved in flavone biosynthesis in Scutellaria baicalensis. Bioresour. Technol., 101: 9715-9722.

DOI: 10.1016/j.biortech.2010.07.083 